\title{
Experiencias educativas de niñas, niños y adolescentes chilenos confinados por la pandemia COVID-19
}

\author{
Tania Ponce Mancilla ${ }^{1}$ (D) ; Constanza Vielma Hurtado ${ }^{2}$ (D) ; Cristián Bellei Carvacho ${ }^{1}$ (1) \\ ${ }^{1}$ Universidad de Chile
}

Resumen. Este artículo reflexiona acerca de las experiencias educativas del aprendizaje en casa de niñas, niños y adolescentes confinados por la pandemia COVID-19 en Chile. A partir de los datos de una encuesta en línea dirigida a madres, padres y apoderados de menores entre 4 y 18 años, aplicada durante el mes de septiembre del 2020 ( $N=4.912)$, se evaluaron las condiciones de educabilidad, sociales y de aprendizaje de los escolares y sus familias en este contexto de crisis. El análisis de este estudio amplía la definición de lo que se entiende por experiencia educativa, reconociendo que las oportunidades de aprendizaje en casa incluyen también las experiencias no formales experimentadas en la convivencia diaria y la vida cotidiana. Los resultados señalan una fuerte profundización de las desigualdades socioeconómicas, evidenciando los desafíos de equidad que aún mantiene pendientes la educación remota. En las reflexiones finales del artículo exploramos las posibilidades futuras de la "escuela pandémica", la importancia del rol de la escuela como espacio de socialización y la fragilidad de esta experiencia vital para niñas y niños.

Palabras clave: pandemia COVID-19; experiencias educativas en casa; desigualdades educativas.

Experiências educacionais de crianças e adolescentes chilenos isolados pela pandemia COVID-19

Resumo. Este artigo reflete sobre as experiências educacionais de aprendizagem em casa de crianças e adolescentes isolados pela pandemia da COVID-19 no Chile. A partir dos dados de uma pesquisa on-line dirigida a mães, pais e responsáveis de crianças entre 4 e 18 anos de idade, aplicada durante o mês de setembro de 2020 (N=4,912), foram avaliadas as condições de educabilidade, sociais e de aprendizagem das crianças em idade escolar e de suas famílias neste contexto de crise. A análise deste estudo amplia a definição do que se entende por experiência educacional, reconhecendo que as oportunidades de aprendizagem em casa também incluem as experiências não formais vividas na convivência diária e na vida cotidiana. Os resultados apontam para um forte aprofundamento das desigualdades socioeconômicas, evidenciando os desafios de equidade que a educação remota ainda enfrenta. Nas reflexões finais do artigo, exploramos as possibilidades futuras da "escola pandêmica", a importância do papel da escola como espaço de socialização e a fragilidade desta experiência vital para meninas e meninos.

Palavras-chave: pandemia COVID-19; experiências educacionais em casa; desigualdades educacionais.

\section{Educational experiences of Chilean children and adolescents confined by the COVID-19 pandemic}

Abstract. This article reflects on the educational experiences of learning at home for children and adolescents confined by the COVID-19 pandemic in Chile. From the data of an online survey directed to parents and guardians of minors between 4 to 18 years old, during the month of September 2020 ( $N=4,912)$, the educability, social and learning conditions of schoolchildren and their families in this context of crisis were evaluated. The analysis of this study broadens the definition of what is understood by educational experience, recognizing that learning opportunities at home also include non-formal experiences experienced in daily living and everyday life. The results indicate a strong deepening of socioeconomic inequalities, showing the challenges that remote education still has pending. In the final reflections of the article, we explore the future possibilities of the "pandemic school", the importance of the role of the school as a space for socialization and the fragility of this vital experience for girls and boys. Keywords: COVID-19 pandemic; educational experiences at home; educational inequalities. 


\section{Introducción}

Una de las medidas inmediatas para enfrentar la propagación de la pandemia por la COVID-19 ha sido el cierre de las escuelas a nivel mundial. En el peak del confinamiento los establecimientos escolares fueron cerrados para más del $90 \%$ de los estudiantes del mundo, dejando a un tercio de niñas y niños completamente privados de educación (UNICEF, 2020). Esta no es una realidad nueva, experiencias de otras crisis sanitarias del pasado reciente -como el ébola o la influenza por H1N1- han demostrado que el mayor impacto de la pandemia en niñas y niños no se relaciona con las tasas de infección, sino que, con las pérdidas en servicios de educación, salud y alimentación por el cierre de las escuelas, aumentando profundamente sus vulnerabilidades (UNICEF, 2015).

En Chile, el escenario no fue diferente. A mediados de marzo del 2020, las autoridades declaran el cierre tanto de las escuelas públicas como privadas -con y sin subvención-, y de los jardines infantiles, coincidiendo con el inicio del año escolar ${ }^{1}$, generando que miles de estudiantes no hayan asistido a clases durante todo ese año. Los efectos a nivel educacional, social y emocional han sido gigantescos para niñas y niños, ya que esta situación inédita y compleja de aprendizaje en casa, ha visibilizado la realidad muchas veces precaria de la educación en Chile.

Este artículo reflexiona acerca de las desigualdades e inequidades en las experiencias educativas de niñas, niños y adolescentes chilenos producidas por la pandemia COVID-19 y la educación en casa. El contexto actual ha obligado a repensar urgentemente qué entendemos por educación, para este efecto, hemos adoptado una amplia definición de "experiencias educativas", la cual comprende que en el aprendizaje no solo influye lo cognitivo, sino que también la realidad material (hogar y sus recursos), social (familia, amigos, conectividad con la escuela) y personal (bienestar socioemocional del niño o niña).

\section{Antecedentes}

La literatura existente sobre otras crisis sanitarias mundiales, han revelado que el cierre de las escuelas tiene fuertes costos económicos y sociales en las familias en torno a su seguridad laboral, ya que los padres deben ausentarse en sus trabajos, y pasar al cuidado de los niños y personas dependientes en el hogar, y a la interrupción de beneficios sociales de niñas y niños más vulnerables, como los programas de alimentación o participación en actividades sociales extraeducativas (Cauchemez, et al., 2009). A su vez, ya se ha investigado acerca de las potenciales pérdidas acumulativas en los estudiantes a lo largo de su trayectorias vitales -producto de las pérdidas de aprendizaje- por el cierre de las escuelas; en términos generales, cada año escolar cursado se asocia con un promedio de ingresos un 10\% más alto en muchos países, por lo que estas estimaciones de pérdida generalizada en los alumnos impactarán más fuertemente en los hogares de los estudiantes más desfavorecidos (Hanusheck y Woessmann, 2020). Esta crisis de los ingresos podría llevar a las familias a poner a trabajar a sus hijos, y puede que muchos nunca regresen a la escuela (Azevedo, et.al, 2020); en las experiencias pasadas en regiones donde las catástrofes fueron

\footnotetext{
${ }^{1}$ Recuperado de https://www.minsal.cl/presidente-anuncia-suspension-de-clases-y-reduce-actos-publicos/.
} 
más intensas, se demostró que niñas y niños vulnerables están más propensos a la desnutrición, y aumentó a casi al doble la proporción de niños matriculados en la escuela y que simultáneamente trabajaban (Baez y Santos, 2007).

Los sistemas educativos del mundo ya venían enfrentando pre-pandemia una crisis de aprendizaje en relación con las desigualdades educativas, el 53\% de las niñas y niños en países de bajos y medianos ingresos eran incapaces de leer y entender un texto simple a la edad de 10 años (Azevedo, et al., 2020), mientras que según datos de UNESCO (2017), unos 263 millones de niños y jóvenes de edades entre 6 y 17 años -la mayoría de ellas niñas-, no asisten a la escuela.

Ahora bien, durante la pandemia ya se ha advertido sobre los riesgos de segregación y desigualdad en las oportunidades de aprendizaje en los hogares, por ejemplo, UNESCO (2020) identificó que los padres, con educación y recursos limitados, no están preparados para la educación a distancia, además de tener que dejar solos a sus hijo/as si es que tienen que trabajar, aumentando la brecha en el cuidado de niñas y niños. En ese sentido, la desigualdad social y educativa, durante esta pandemia, se reproduce y amplía a partir del capital cultural de las familias (Tarabini, 2020; Dietrich, Patzina y Lerche, 2021), y más aún en los hogares monoparentales (Cabrera, Pérez y Santana, 2020). Además, las familias han declarado tener un bajo nivel de conocimiento de enseñanza virtual, ya que vinculan el uso de tecnologías principalmente con el ocio y el divertimento (Hortigüela-Alcalá, et al., 2020); y a esto se le suma, que ahora los padres perciben que tienen la responsabilidad principal de que sus hijos sigan aprendiendo en casa, "obligados a hacer malabarismos con sus propias luchas personales y profesionales" (Fontenelle-Tereshchuk, 2021).

Las desigualdades no sólo son por clase social, el género también es una variable determinante. Ya existe evidencia de que, al cerrarse las escuelas, las niñas sufrieron un aumento en las responsabilidades domésticas y de cuidados, a esto se le suma que están más expuestas al abuso físico y sexual (Giannini y Albrectsen, 2020).

En términos de bienestar socioemocional, estudios demuestran que en tiempos de adversidad, los estudiantes pueden mostrar diferentes reacciones hacia los demás: algunos necesitarán mayor necesidad de afecto, apoyo social y tranquilidad, mientras que otros -sobretodo en un contexto de pérdida de seres queridos- se alejarán de sus compañeros y maestros (Tanyu, et al. , 2020). Un estudio² sobre jóvenes de entre 14-18 años en Ecuador, reveló que el 16\% de los estudiantes tiene depresión mayor, y que los niveles de ansiedad y estrés son menores en estudiantes de hogares más ricos, con acceso a Internet y madres más educadas (Asanov, et al., 2020). Otro estudio ${ }^{3}$, demostró que 1 de cada 5 estudiantes tenían problemas emocionales o de conducta, y estudiantes de bajo grados fueron considerados más vulnerables (Zhao, et al.,

\footnotetext{
2 Encuesta telefónica de respuesta rápida a 1500 estudiantes de entre 14-18 años, que buscaba medir cómo utilizaban su tiempo los estudiantes durante la cuarentena, examinar su acceso a aprendizaje remoto y medir su estado de salud mental.

${ }^{3}$ Los comportamientos y sentimientos de educación en el hogar de los niños en edad escolar se evaluaron con encuestas en línea de 2010 obtenidas por separado de estudiantes, padres y maestros de los grados 1 a 9 en 15 provincias chinas. Las respuestas fueron comparadas entre los grupos de grado bajo (grados 1-3), medio (grados 4-6) y alto (grados 7-9). ( $N=738$ respondidos por estudiantes, 1062 respondidas por padres (o tutores similares) y 210 respondidas por maestros).
} 
2020), sin embargo, hay evidencia que demuestra que respecto a lo socioemocional no hay diferencia por niveles educacionales, incluso llegando los jóvenes mayores a necesitar mayor apoyo emocional (Fontenelle-Tereshchuk, 2020).

Investigaciones han determinado la importancia de la actividad física en la salud corporal y psicosocial de niña/os y jóvenes (Roe, et al., 2021), situación que durante esta pandemia se ha visto afectada y en descenso, aumentando el uso de pantallas y del sedentarismo o baja actividad física de niñas y niños (Bonal y González, 2020), debido a la falta de espacio y disponibilidad de herramientas para deportes organizados (Carroll, et al., 2020), estando considerablemente menos activos que cuando estaba la escuela abierta.

Este periodo de aprendizaje en casa, también ha visibilizado la complejidad en el acceso a recursos educativos, un estudio mundial ${ }^{4}$ dio cuenta que este no es justo ni equitativo, hubo casi un $10 \%$ de estudiantes que no accedió a trabajos con textos, libros y educación online impartida por profesores (Reimers y Schleicher, 2020). Más desigual es el acceso a tecnología durante la pandemia en los distintos grupos de estudiantes, no todos los niños pueden participar en sus actividades básicas digitalizadas, pues poseen problemas con el acceso a Internet, los dispositivos y las aplicaciones (livari, et al., 2020), además de que no todos comprenden el uso de estas; esto a visibilizado una nueva forma de exclusión, basado en la educación (los que saben o no) y en el acceso (los que tienen o no) (Van der Vlies, 2020). Adicionalmente, se podría afirmar que las desigualdades en el aprendizaje en casa se ven afectadas por las condiciones de las viviendas en torno a su bienestar, ya que éstas están fuertemente relacionadas con la clase social o ingreso socioeconómico (Filandri y Olagnero, 2014), evidenciando diferencias en los esfuerzos escolares de niñas y niños que tienen acceso a una habitación propia y tranquila, respecto a los que no (Dietrich, Patzina y Lerche, 2021).

El acceso a recursos educativos varía considerablemente por tipo de escuela, la evidencia mundial muestra que las escuelas privadas entregan más clases en línea que las escuelas públicas, encontrándose desigualdades dentro de este sector (Andrew, et al., 2020), durante la pandemia un número significativo de escuelas públicas no desarrollaron tareas escolares, mientras las escuelas concertadas y privadas no detuvieron su actividad lectiva (Bonal y González, 2020).

\section{Métodos y datos}

El presente estudio tiene un carácter transversal y cuantitativo. El análisis cuantitativo se realizó con los datos obtenidos por medio de un cuestionario estructurado, usando cruces estadísticos y regresión múltiple para las variables de mayor relevancia.

La encuesta Experiencias educativas en casa de niñas y niños durante la pandemia covid-19 estuvo disponible entre el 1 de septiembre y el 14 de octubre del 2020. Se realizó un cuestionario online basado parcialmente en la encuesta desarrollada por la Universidad Autónoma de Barcelona "Confinamiento y Condiciones de Aprendizaje" (Bonal y González, 2020).

\footnotetext{
${ }^{4}$ Estudio elaborado a partir de la participación de 59 países. Se les aplicó una encuesta online a distintos representantes de la comunidad escolar entre el 25 de abril y el 7 de mayo $(\mathrm{N}=1.370)$.
} 
Los informantes fueron madres, padres o tutores de menores entre 4 y 18 años que cursaban algún nivel de escolaridad obligatoria en cada una de las comunas de Chile. Según los datos del Mineduc, a abril del 2020, la cantidad de menores matriculados en el país ascendía a 3.608.158 niños, niñas y adolescentes (Mineduc, 2020). Los niveles educativos del sistema escolar en Chile se dividen en tres, educación preescolar (4 y 5 años), básica (6 a 13 años) y media (14 a 17 años), de los cuales sólo "Educación básica" y “Educación media" son obligatorios.

Otra particularidad del sistema educativo chileno es la variedad en el tipo de escuela disponible para los estudiantes. Para efectos de este artículo se nombrarán de acuerdo a su propiedad (público/privada) y financiamiento (gratuita/subsidiada/ pagada). Si bien las escuelas públicas son, por definición, gratuitas ${ }^{5}$, las escuelas privadas pueden recibir o no financiamiento por parte del Estado, y exigir o no el pago de aranceles. Por lo tanto, hablaremos de: i) escuelas públicas; ii) privadas subvencionadas por el Estado y gratuitas; iii) privadas subvencionadas por el Estado y pagadas por las familias; $y$, iv) privadas financiadas únicamente mediante el pago de las familias.

\subsection{Diseño muestral}

Para la selección final de la muestra se utilizó un muestreo no probabilístico por cuotas (tomando como base una cobertura en las 16 regiones del país). Es importante señalar que no se esperaba tener casos para todas las comunas dado el sesgo de conectividad y la menor probabilidad de alcance de la encuesta en municipios muy rurales o con muy poca matrícula. Sin embargo, para ajustar los datos a las particularidades geográficas y socio demográficas locales, se optó por realizar de forma posterior a la recogida de datos un proceso de ponderación post estratificación según comuna de residencia del hogar y dependencia administrativa de la escuela a la que asiste el o la menor.

Se alcanzó una muestra de 4.912 hogares con menores en edad escolar, con casos en todas las regiones del país, representando zonas donde se ubica un $87 \%$ de la matrícula nacional.

\subsection{Caracterización de la muestra.}

La mayor cantidad de personas que contestaron la encuesta se identifican con el género femenino (87\%) mientras que sólo un $13 \%$ con el género masculino. De este grupo, la mayoría de las encuestadas declararon ser las madres de las niñas y niños $(76,5 \%)$.

Con respecto al género con el que identificaron a la o el menor, se observa una muestra equilibrada, ya que el $51,7 \%$ declaró pertenencia al género femenino, frente a un $48,2 \%$ al masculino. Los menores no binarios alcanzan un $0,04 \%$ de la muestra ${ }^{6}$. La edad más frecuente de las y los menores de la muestra recogida fue de $8(10 \%)$, 5 (9\%) y 12 años (8\%). La edad menos frecuente fue de 18 años (3\% de la muestra).

\footnotetext{
${ }^{5}$ Escuelas públicas incluyen a las escuelas Municipales y Servicios Locales de Educación.

${ }^{6}$ Menores con género "No binario" no se incluyeron en cruces de otras tablas por género a causa del tamaño pequeño de casos $(\mathrm{N}<5)$.
} 
El máximo nivel educacional completado por la madre en la muestra fue, con mayor frecuencia, la educación universitaria (44\%), seguida por la educación secundaria completa (25\%). Una menor proporción de las madres alcanzaron el nivel de postgrado (14\%) o completaron la educación terciaria técnico-profesional (11\%). Finalmente, sólo un 7\% de las madres no terminaron la educación secundaria.

Antes de la pandemia, un $17 \%$ de los hogares de la muestra percibían ingresos mensuales de 556 USD o menos, y, en su mayor parte entre 557 y 1.113 USD (22\%), seguidos por el grupo que percibía entre 1.114 y 1.670 USD (21\%). Esto quiere decir que un $60 \%$ de los hogares de la muestra percibían menos de 1.670 USD. Por otro lado, un $16 \%$ de los hogares percibieron entre 1.670 y 2.227 USD mensuales, y en un menor porcentaje los encuestados reportaron ingresos mensuales entre 2.227 y 3.480 USD, y/o más de 3.480 USD (13\% y $11 \%$, respectivamente), siendo muy similar a la reportada por el Instituto Nacional de Estadísticas de Chile.

\section{Resultados}

\subsection{La difícil producción de experiencias educativas en casa para escuelas y docentes}

En términos generales, las escuelas parecen haber reaccionado de manera rápida a la interrupción de clases presenciales: al mes de abril el $70 \%$ de las familias ya habían tomado contacto con las escuelas. Aunque en algunos casos este contacto fue decreciendo en el tiempo o fue intermitente, al momento de responder la encuesta (septiembre) el 63\% de las familias afirmó que el contacto ha sido permanente durante la pandemia. Aquí ya se observan desigualdades: mientras un 55\% de los padres o madres de estudiantes de escuelas públicas reportó un contacto permanente entre la escuela y el alumno, esta proporción asciende a un $80 \%$ en escuelas privadas.

Ciertamente, la calidad y versatilidad de la comunicación establecida entre la escuela y los hogares de los estudiantes depende del medio que se utilice. En este aspecto, la posibilidad de realizar clases online constituye el reemplazo más cercano de la clase presencial. De acuerdo a los resultados, sólo un $40 \%$ de los estudiantes recibe clases online todos los días, existiendo en este aspecto una desigualdad socioeconómica relacionada con el tipo de escuela al que se asiste: mientras el $80 \%$ de estudiantes de escuelas privadas tiene clases online diariamente; un 30\% niños y niñas de escuelas públicas y subvencionadas gratuitas accede a clases todos los días; y más aún, un 18\% de los alumnos de escuelas públicas declara "nunca" tener clases online. Debe recordarse que los estudiantes con peor conectividad no están bien representados en la muestra, por lo que estas diferencias pueden ser mayores en el total de la población escolar.

Como compensación por la falta de intensidad del soporte vía clases online las escuelas están usando variadas estrategias: la más recurrida son las redes sociales (WhatsApp, Instagram), más comunes en las escuelas públicas (65\%) que en las privadas $(30 \%)$, pero incluso medios más tradicionales como las llamadas telefónicas, son importantes en las escuelas públicas (42\%), mientras son marginales en las escuelas privadas $(7 \%)$. 
El uso de guías y textos de estudio, ha sido otro medio de enseñanza muy intenso: un $86 \%$ del total de alumnos trabaja con ellas "todos" o "algunos" días a la semana. El 85\% de las escuelas privadas envía el material de estudio de forma online todas las semanas, contra un $55 \%$ en escuelas públicas. Alrededor de un tercio de las familias de escuelas públicas ha ido a retirar guías y otros materiales de manera presencial a las escuelas al menos algunas veces al mes, sin embargo, esta proporción desciende a un 3\% para los que asisten a escuelas privadas. Ver videos preparados o recomendados por los profesores es una actividad masivamente extendida de manera transversal para los alumnos de todas las escuelas, alcanzando un $78 \%$ de los alumnos y alumnas en escuelas públicas y un $90 \%$ en escuelas privadas que dicen hacerlo "todos" o "algunos" días a la semana.

Gráfico 1. Actividades educativas realizadas todos los días o algunos días a la semana por estudiantes, en porcentaje, por tipo de escuela.

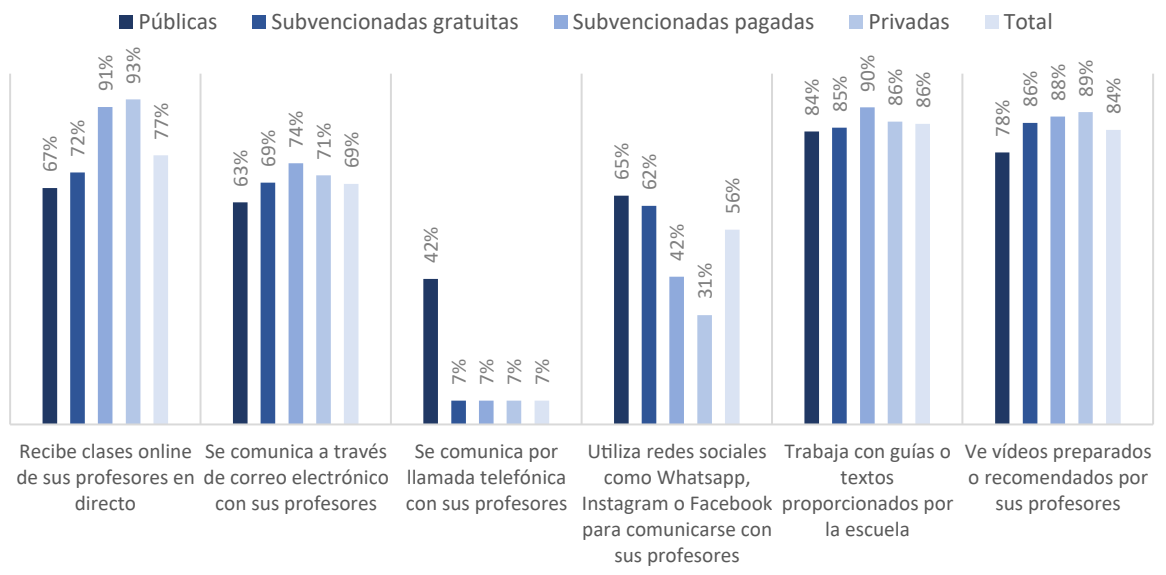

Nota: Resultados ponderados. $(\mathrm{N}=4.285)$

Fuente: Elaboración propia a partir de base de datos "Experiencias educativas en casa de niñas y niños durante la pandemia COVID-19" CIAE-UCHILE

Por último, en relación al tipo de actividades que las escuelas promueven entre sus estudiantes, hay una gran intensidad y muy universal uso de tareas/ejercicios, a saber: alrededor de un $80 \%$ de los estudiantes recibe tareas para hacer solos "todos los días" o "algunos días a la semana". Es interesante que una proporción mayoritaria $(60 \%)$ recibe también tareas para hacerlas con su familia al menos semanalmente, lo cual es más común entre los preescolares (80\%) que en la secundaria alta ${ }^{7}(50 \%)$. En contraste, el recurso de dar tareas para hacerse con compañeros/as es muy poco utilizado: al $70 \%$ de estudiantes de primaria/secundaria baja y $45 \%$ de secundaria alta "nunca" les han hecho trabajar con sus pares durante este año. Sin embargo, hay diferencias por tipo de escuela, siendo más de dos veces más frecuente el trabajo con pares "al menos algunas veces a la semana" en escuelas privadas (30\%) que en escuelas públicas (13\%),

\footnotetext{
${ }^{7}$ En Chile los niveles de escolaridad equivalentes son: de $1^{\circ}$ a $6^{\circ}$ básico (primaria), $7^{\circ}$ y $8^{\circ}$ básico (secundaria baja) y de $1^{\circ}$ a $4^{\circ}$ medio (secundaria alta).
} 


\subsection{Hogar: ¿Cuál es el contexto de la escuela pandémica?}

La familia siempre ha sido una mediadora entre la escuela y los estudiantes, pero nunca tan decisiva como hoy. Ciertamente, la casa no es la escuela, pero ha estado emulándola. Para ser provista como un derecho igualitario de niños y niñas, la educación formal, ha sido organizada alejando lo más posible las extremadamente desiguales condiciones familiares de origen de los estudiantes, y ofreciendo, a cambio, una experiencia común en tiempo, recursos, currículum, espacio de sociabilidad y estudio. Confinar la escuela en el hogar arriesga sensiblemente este principio elemental de equidad.

La encuesta muestra proporciones muy importantes de familias afectadas seriamente en términos de salud como económicos, lo que constituye el contexto vital de los estudiantes. La mayoría de las personas (63\%) declaró que sus familias habían vivido alguna situación crítica en el hogar durante la pandemia: el 23\% reportó la muerte de algún familiar o amiga/o cercana; el $26 \%$ la pérdida del trabajo remunerado; un 15\% problemas económicos graves; y un $6 \%$ afirmó que al menos una persona en el hogar se había enfermado de coronavirus. Mientras un 53\% de las familias del quintil socioeconómico 5 (mayores ingresos) reportaron una situación crítica en el hogar durante la pandemia, esta proporción aumentó a un 70\% entre los hogares del quintil 1 , de menores ingresos.

Gráfico 2. Porcentaje de hogares cuyos integrantes han vivido una o más situaciones críticas durante el confinamiento, por quintil de ingresos

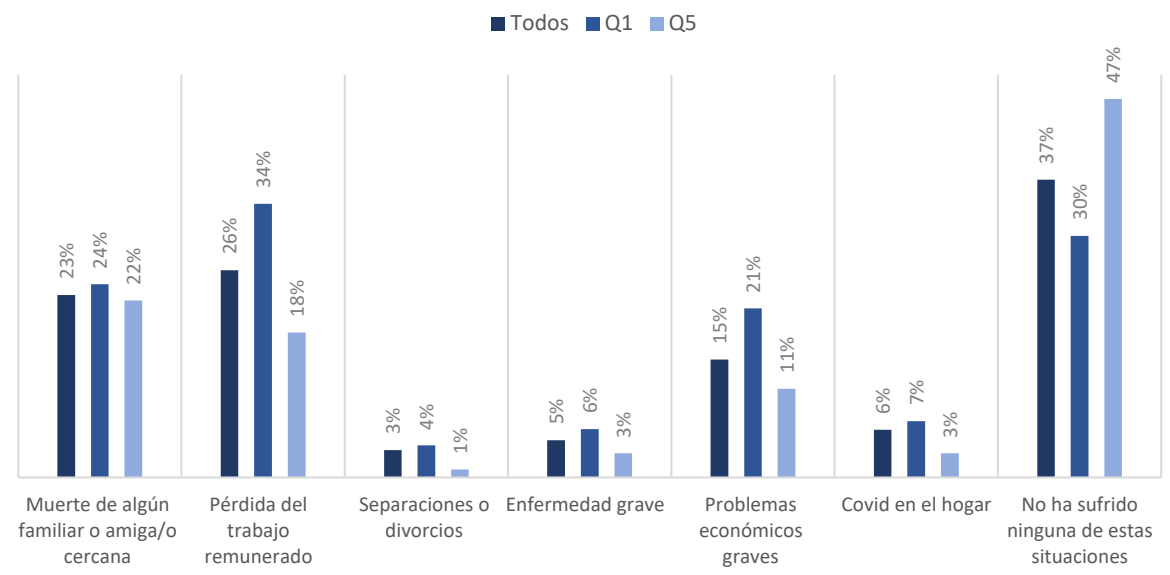

Nota: Resultados ponderados. ( $\mathrm{N}=4.912)$

Fuente: Elaboración propia a partir de base de datos "Experiencias educativas en casa de niñas y niños durante la pandemia COVID-19" CIAE-UCHILE

También existieron repercusiones de la pandemia en las desigualdades dentro del núcleo familiar en términos de género. Por ejemplo, la mitad de las personas declaró estar totalmente de acuerdo o de acuerdo en que, durante el confinamiento, niñas y mujeres han estado más expuestas a algún tipo de violencia en el hogar (psicológica, física, sexual), mientras el $71 \%$ afirmó que las mujeres (adultas y niñas) se han hecho mayormente cargo de las tareas domésticas y de cuidados del hogar. 
Además de su relevancia intrínseca, esto tiene un impacto directo en el proceso de aprendizaje de los estudiantes, pues han sido las madres el principal apoyo para las actividades escolares de niños y niñas en casa, haciéndolo siempre o la mayoría de las veces $(77 \%)$, lo que contrasta fuertemente con el nivel de apoyo provisto por el padre (26\% siempre o a veces).

Ahora bien, ya que la casa acoge la escuela, la materialidad y el equipamiento de la vivienda se vuelven críticos para el proceso de aprendizaje. La mitad de las familias vive en viviendas de 80 metros cuadrados o menos, lo que impacta directamente en el espacio para estudiar. Por ejemplo, en viviendas con más de 100 mts. cuadrados, más del $80 \%$ tiene siempre un espacio propio o habitación donde estudiar y hacer las tareas. Por el contrario, en los hogares de hasta 30 mts. cuadrados, sólo el $36 \%$ tiene siempre un espacio donde estudiar, mientras el $34 \%$ no lo posee nunca o casi nunca. Más fundamental en este período: un tercio de las familias no cuenta en sus viviendas con un espacio exterior de uso exclusivo; proporción que aumenta a más de la mitad (53\%) en el quintil uno, y desciende a cerca de un quinto (22\%) en el quintil 5.

Gráfico 3. Porcentaje de niñas y niños de la muestra que cuenta con recursos y espacios educativos en su hogar, según dependencia administrativa del establecimiento al que asisten (\% siempre).

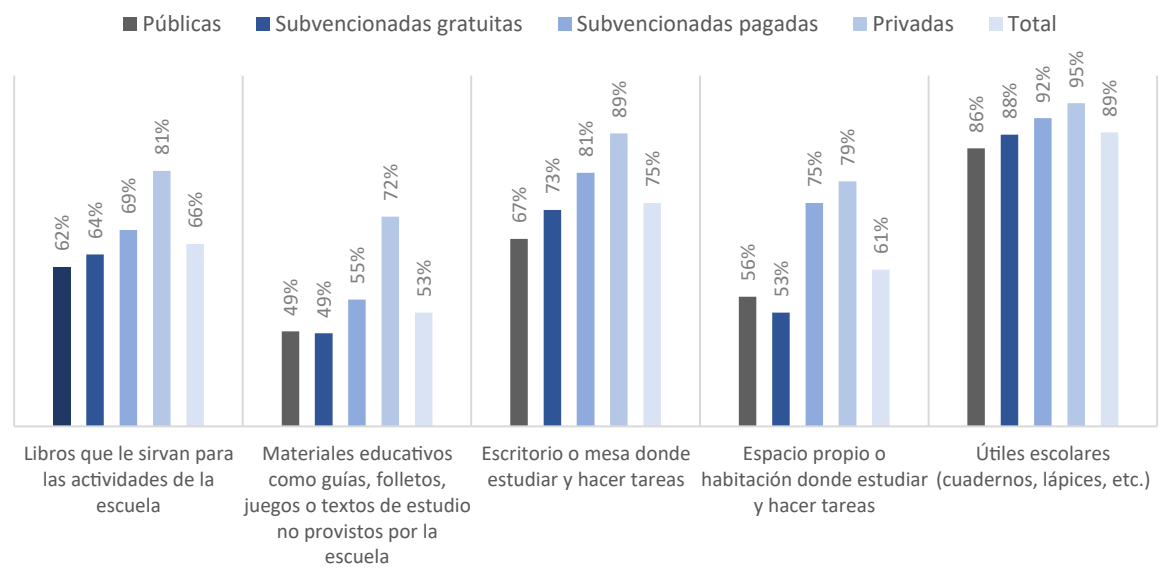

Nota: Resultados ponderados. $(\mathrm{N}=4.285)$

Fuente: Elaboración propia a partir de base de datos "Experiencias educativas en casa de niñas y niños durante la pandemia COVID-19" CIAE-UCHILE

En general, los hogares encuestados también tienen acceso a recursos educativos más tradicionales como libros de apoyo, una mesa o escritorio y un espacio propio o habitación donde estudiar. Sin embargo, mientras la gran mayoría (en torno al $80 \%$ ) de quienes asisten a escuelas privadas "siempre" cuentan con estos recursos educativos en su hogar, entre los estudiantes de escuelas públicas y subvencionadas gratuitas estos recursos están "siempre" disponibles para un 50\% y 70\%, dependiendo del recurso que se trate. Una habitación o espacio propio para hacer tareas y estudiar 
es el recurso más limitado para los estudiantes más vulnerables: un $25 \%$ no tiene acceso "nunca" o solo "a veces" a un espacio propio, situación que sólo viven un 10\% de los niños y niñas de escuelas privadas.

Por último, para que la escuela pandémica se active en casa, los dispositivos tecnológicos con capacidad de conexión a internet se vuelven esenciales. En este punto se debe recordar que la encuesta se auto-aplicó online, por tanto, no representa a las familias que no poseen estos medios al menos en grado básico. Así, la disponibilidad de ordenador o tablet en los hogares encuestados es alta: más del $90 \%$ cuenta con al menos uno y el $60 \%$, con 2 o más ordenadores portátiles. Sin embargo, solo 1 de cada 2 niños cuenta con un ordenador (de cualquier tipo) o tablet de uso exclusivo para su trabajo escolar; siendo las desigualdades sociales muy marcadas: mientras casi un $70 \%$ alumnos de escuelas privadas cuentan con un ordenador exclusivo para su trabajo escolar, entre sus pares de escuelas subvencionadas gratuitas, sólo un $36 \%$ tiene este recurso, y un $12 \%$ de los alumnos de las escuelas gratuitas no cuenta con ordenador en el hogar.

Gráfico 4. Porcentaje de niñas y niños de la muestra que cuenta con recursos tecnológicos en su hogar según dependencia administrativa.

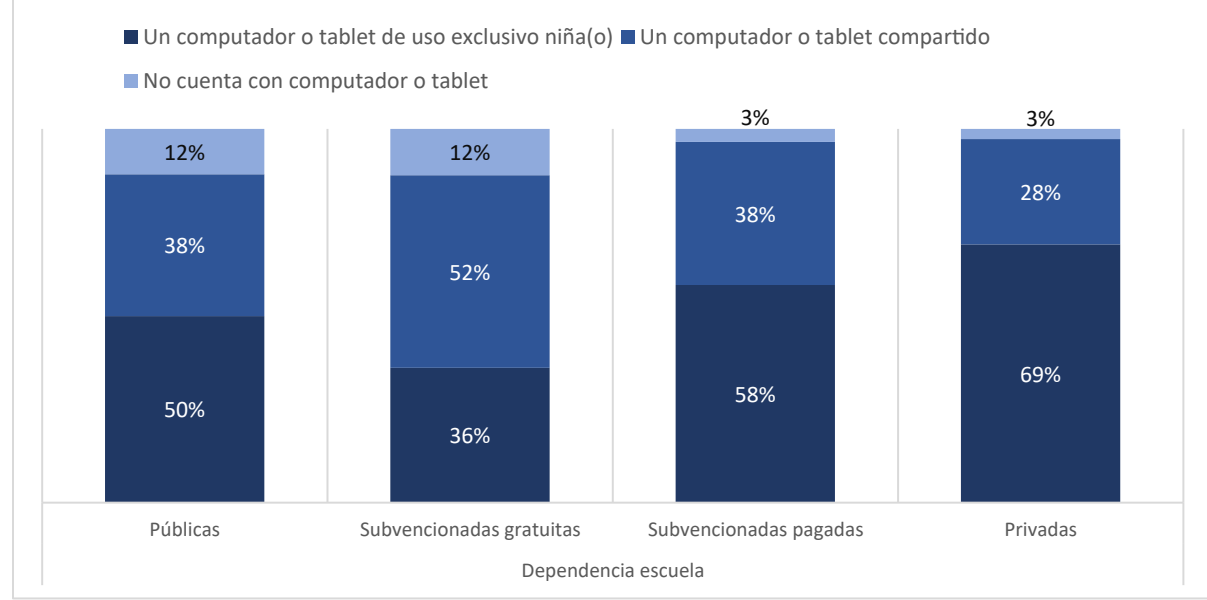

Nota: Resultados ponderados. $(\mathrm{N}=4.011)$

Fuente: Elaboración propia a partir de base de datos "Experiencias educativas en casa de niñas y niños durante la pandemia COVID-19" CIAE-UCHILE

La conectividad es vital para la enseñanza remota. Un $60 \%$ de los hogares tiene conexión fija (banda ancha, cable o fibra óptica), y un 33\% tiene algún tipo de conexión móvil. Al evaluar la calidad de la conexión a internet para permitir las actividades escolares, sólo el $37 \%$ la considera óptima; otro tercio considera que, aunque "es rápida, se desconecta a veces"; y un cuarto de los hogares reporta una conexión deficiente para el trabajo escolar. Las desigualdades sociales son también marcadas: casi un tercio (29\%) de quienes asisten a escuelas gratuitas (públicas y subvencionadas) considera su conexión deficiente, juicio que sólo tiene el $14 \%$ de quienes asisten a escuelas privadas. Estas desiguales condiciones en el hogar se encuentran probablemente asociadas al acceso a clases online mencionado. 
Gráfico 5. Porcentaje de hogares de la muestra por tipo de conexión a internet, y calidad de la conexión, según tipo de escuela.

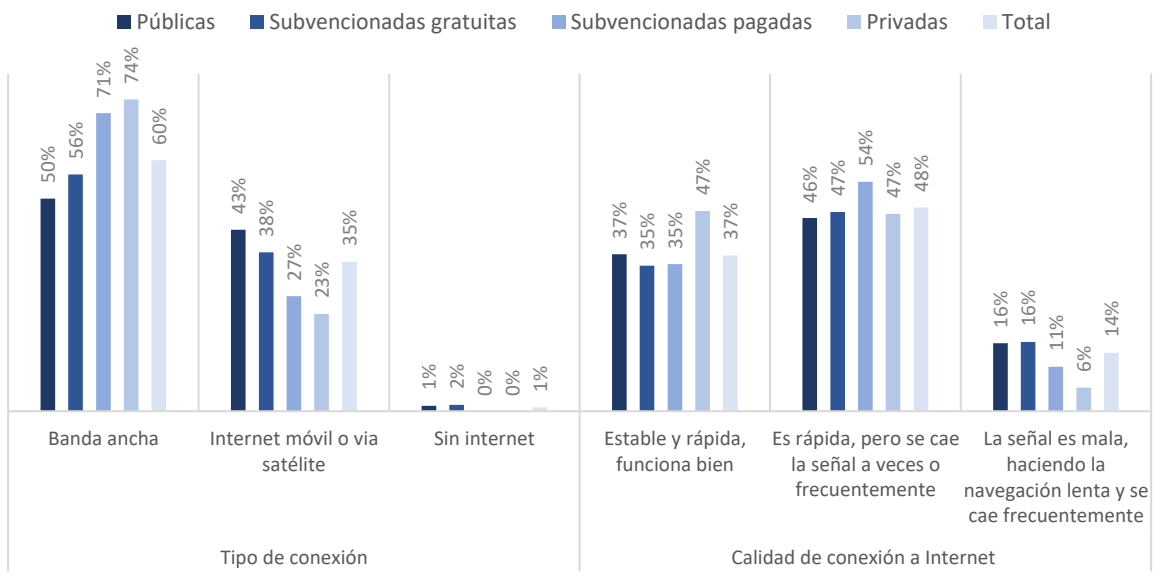

Nota: Resultados ponderados. $(\mathrm{N}=4.013)$

Fuente: Elaboración propia a partir de base de datos "Experiencias educativas en casa de niñas y niños durante la pandemia COVID-19" CIAE-UCHILE

\subsection{Estudiantes: La experiencia de la escuela pandémica en casa}

Con respecto a la apreciación de la carga de trabajo que las escuelas han puesto sobre los estudiantes, las familias se dividen casi en dos: alrededor del 40\% estima que ésta ha sido suficiente y otro $40 \%$, que ha sido bastante o excesiva (incluso en educación preescolar, un $25 \%$ de los padres lo declaró). También existen aquí diferencias por escuela: mientras un $6 \%$ de las madres, padres y tutores de escuelas privadas calificó la carga de trabajo como "Insuficiente", esta proporción asciende a un $17 \%$ en escuelas públicas, mientras que la carga fue calificada como excesiva en mayor porcentaje en las escuelas subvencionadas pagadas (20\%).

Debido a la permanencia extendida de los niños y niñas en sus hogares, la encuesta indagó con detalle sobre las diferentes actividades que están realizando en este periodo, más allá de aquellas directamente vinculadas al proceso escolar. Así pudimos observar que el confinamiento ha posibilitado que niñas y niños exploren nuevas formas de aprendizaje en casa, desarrollando actividades creativas, deportivas, de ocio y sociabilidad; algunas actividades transversales a todos los sectores sociales y otros con mayor desigualdad. Por ejemplo, la gran mayoría de las niñas y niños $(86,3 \%)$ están todos los días frente a una pantalla (televisión, tablet o celular), siendo la actividad más universal; además, otras actividades rutinarias que se dan todos los días son ayudar en tareas domésticas en el hogar como limpiar, cocinar y ordenar (40\%), chatear con amigos/as o comunicarse a través de redes sociales (33\%) y leer o jugar videojuegos (32\%). En cambio, ejercicios y actividades deportivas se realizan con muy baja frecuencia, especialmente en el caso de los estudiantes de secundaria alta. 
En esta dimensión nos encontramos con importantes diferencias de género: las niñas realizan mayormente actividades como dibujar, pintar o hacer manualidades, así como colaborar en tareas domésticas y socializar en redes sociales; los niños, en cambio, cotidianamente están jugando videojuegos (72\% todos los días o algunos días por semana, frente a un $45 \%$ de las niñas). Además, el confinamiento no afecta a todos por igual: los niños que viven en hogares sin acceso a espacios exteriores exclusivos "nunca" realizan actividades deportivas y ejercicios $(28 \%)$ que las y los niños que sí tienen acceso (16\%), al igual que "nunca" jugar sin usar pantallas (23\% entre aquellos sin espacio exterior y 14\% aquellos que sí lo poseen).

Gráfico 6. Frecuencia de actividades cotidianas en confinamiento, por género.

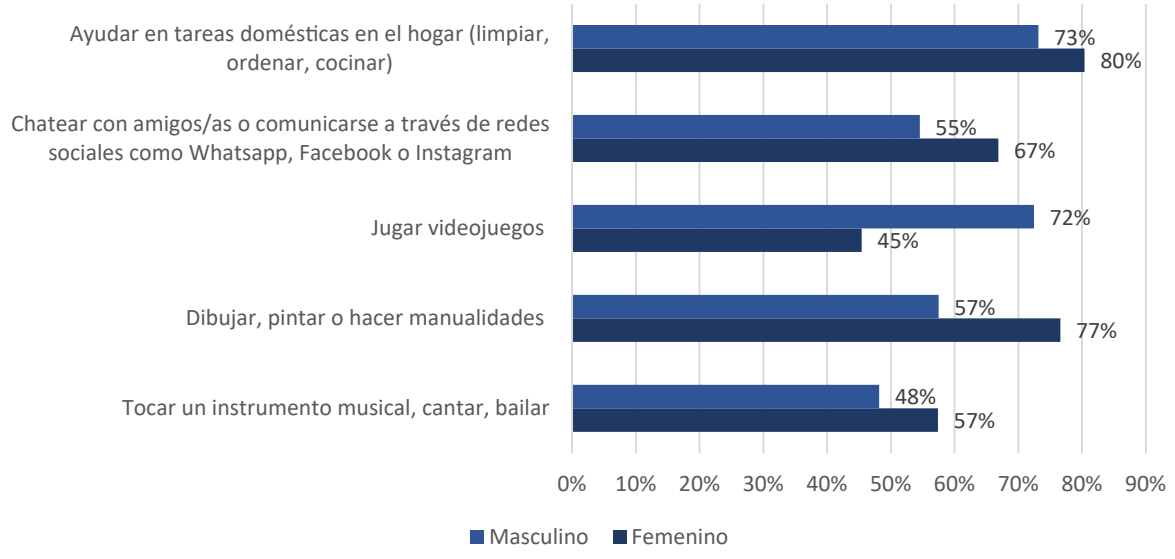

Nota: Resultados ponderados ( $\mathrm{N}=4.124)$.

Fuente: Elaboración propia a partir de base de datos "Experiencias educativas en casa de niñas y niños durante la pandemia COVID-19" CIAE-UCHILE

Por último, con respecto al bienestar socioemocional de las niñas y niños en edad escolar, la encuesta muestra resultados preocupantes, revelando el fuerte y generalizado efecto de esta crisis. Los problemas de mayor incidencia, que se presentan siempre o muchas veces, fueron que se aburren con facilidad (52\%), les cuesta concentrarse (46\%), no quieren hacer las tareas (43\%) y se frustran con frecuencia $(40 \%)$, todas dimensiones que podrían relacionarse con la disposición a actividades escolares. Adicionalmente, problemas más básicos de salud socioemocional, como estar más peleador o irritable, cambios de apetito, no querer levantarse o tener problemas con el sueño, afectan a entre un cuarto y un tercio de niños y niñas.

Los resultados también dieron cuenta que tanto niñas como niños han sido afectados de manera bastante similar a nivel socioemocional. Sin embargo, existen algunas diferencias de género que son interesantes de visibilizar, por ejemplo, en el caso de las niñas, un $29 \%$ no quiere levantarse de la cama "siempre" o "muchas veces", versus un $21 \%$ de los niños. También, en la variable problemas para dormir/ pesadillas, se declara en un $36 \%$ que los niños nunca sufren este evento, versus un $29 \%$ de las niñas (brecha de género de 7 puntos porcentuales). Por su parte, las 
situaciones donde el niño se ve más afectado (niveles siempre y muchas veces), es en las variables falta de concentración y no querer hacer las tareas, donde la brecha de género es de 10 y 11 puntos porcentuales en contra de los niños, respectivamente.

El acceso a espacio exterior de uso exclusivo también presentó cierta asociación con los resultados socioemocionales de los y las niñas: los hallazgos presentan las siguientes incidencias de conductas "siempre o muchas veces" entre aquellos sin acceso a espacio exterior y los que sí lo tienen: Problemas para dormir o pesadillas (30\% y $20 \%$, respectivamente), Cambios de apetito (38\% y $27 \%$, respectivamente), Se aburre con facilidad ( $61 \%$ y $47 \%$, respectivamente), y Está peleador/a, sensible o llora con frecuencia (44\% y $31 \%$, respectivamente) .

Gráfico 7. Porcentaje de madres, padres y tutores que reportaron que niños y niñas presentaron situaciones socioemocionales disruptivas en confinamiento, por tipo de espacio exterior de la vivienda (\% siempre o muchas veces).

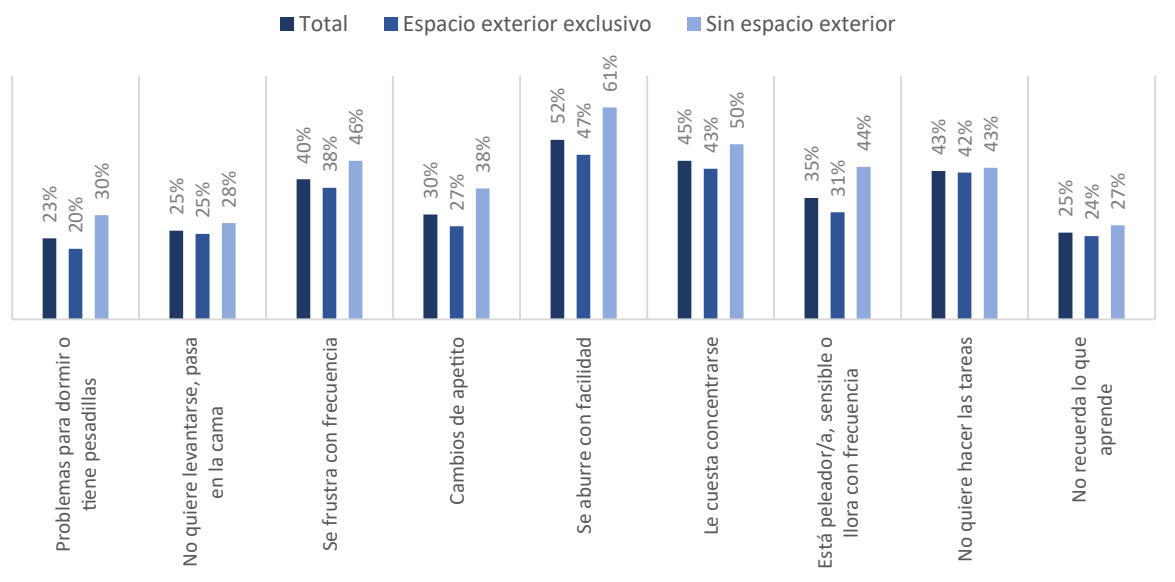

Nota: Resultados ponderados ( $\mathrm{N}=3.123)$.

Fuente: Elaboración propia a partir de base de datos "Experiencias educativas en casa de niñas y niños durante la pandemia COVID-19" CIAE-UCHILE

Finalmente, el indicador fundamental que refleja los contrastes en la manera en que familias y estudiantes gestionan la experiencia de estudiar en casa, es el tiempo escolar. Para orquestar experiencias relevantes de aprendizaje para los estudiantes es necesario activar la motivación y disposición a instruirse, y transformarlas en tiempo de aprendizaje. Dicho esto, existen diferencias importantes entre los estudiantes en cuanto a las horas de dedicación a actividades escolares: mientras el $27 \%$ dedica una hora o menos al día, un tercio (33\%) dedica 4 horas diarias o más. Si proyectamos esta diferencia a un mes de trabajo escolar, encontramos que un grupo de estudiantes ha acumulado, a lo más, 20 horas de estudio en un mes; otros, en cambio, han acumulado ese nivel de estudio en solo una semana, totalizando en el mes, por lo menos, 80 horas. En esto hay grandes brechas por nivel educativo y dependencia del establecimiento, siendo los estudiantes de secundaria alta y de escuelas privadas quienes dedican más horas al aprendizaje. 
La tabla siguiente presenta los resultados de la regresión múltiple usando 3 modelos explicativos de la variable dependiente "Horas diarias dedicadas a actividades escolares". Los resultados están organizados de la siguiente forma: el modelo 1 incluye variables acerca de características del estudiante (sexo, edad) y su contexto escolar: el tipo de escuela y el nivel educativo que cursa. En el modelo 2 se ingresan además variables acerca del contexto del hogar, tales como: quintil de ingreso, acceso a espacio exterior, tipo de conexión a internet y presencia de dispositivos tecnológicos. Finalmente, al modelo 3 se le agregan indicadores referentes a la experiencia en la escuela pandémica del estudiante, estas son: la percepción de la carga escolar, la frecuencia en la que recibe clases online y la presencia de problemas socioemocionales.

La comparación entre modelos del más sencillo al más complejo permite evaluar el aporte de cada bloque de variables a la capacidad predicitiva del modelo mediante el estadístico R2. Este indica que el modelo 1, o las variables relacionadas con características del niño y su escuela, explican alrededor de un $11 \%$ de la varianza de la variable dependiente, mientras que el añadir las variables relacionadas con el hogar suman un $3 \%$ adicional a la capacidad explicativa del modelo. Por último, la inclusión de las variables relacionadas con la experiencia en la escuela pandémica agrega casi un $8 \%$ a la varianza explicada. De esta manera, el modelo completo explica alrededor de un $22 \%$ de la variable "Horas diarias dedicadas a actividades escolares".

Con respecto al efecto de cada una de las variables en el modelo completo, vemos que la mayor parte tienen un efecto estadísticamente significativo, pero con ciertos matices. El ser estudiante mujer aumenta en 0,11 las horas promedio de estudio, en relación a ser hombre, efecto significativo al 90\% de nivel de confianza. El asistir a escuelas públicas o subvencionadas gratuitas disminuye entre 0,2 y 0,5 horas de estudio, en comparación a asistir a una escuela privada. Los accesos a espacio exterior exclusivo, a conexión a internet mediante banda ancha y a un ordenador/tablet de uso exclusivo, aumentaron en $0,13,0,25$ y 0,26 , respectivamente, el número de horas de estudio promedio en relación a no tener acceso a estos recursos en el hogar. Pertenecer a los quintiles 3, 4 y 5 de ingreso aumenta el número de horas de estudio predichas en entre un 0,3 y 0,5, en relación a pertenecer al quintil 1. El evaluar la carga escolar como Suficiente, Bastante o Excesiva, aumenta entre un 0,4 y un 0,9 las horas de estudio, en relación a haber evaluado la carga escolar como Insuficiente; mientras que tener clases online "todos los días" aumenta en 1,2 horas promedio el estudio diario, en relación a "Nunca" tener clases online. Por último, la presencia frecuente de problemas socioemocionales como "Le cuesta concentrarse" y "No quiere hacer las tareas" disminuye las horas predichas de estudio en 0,3 aproximadamente. 
Tabla 1. ¿Qué explica el tiempo de dedicación a actividades escolares en casa? Modelos de regresión múltiple que asocian las "Horas diarias dedicadas a actividades escolares" (variable dependiente) con diferentes características de los estudiantes y sus hogares.

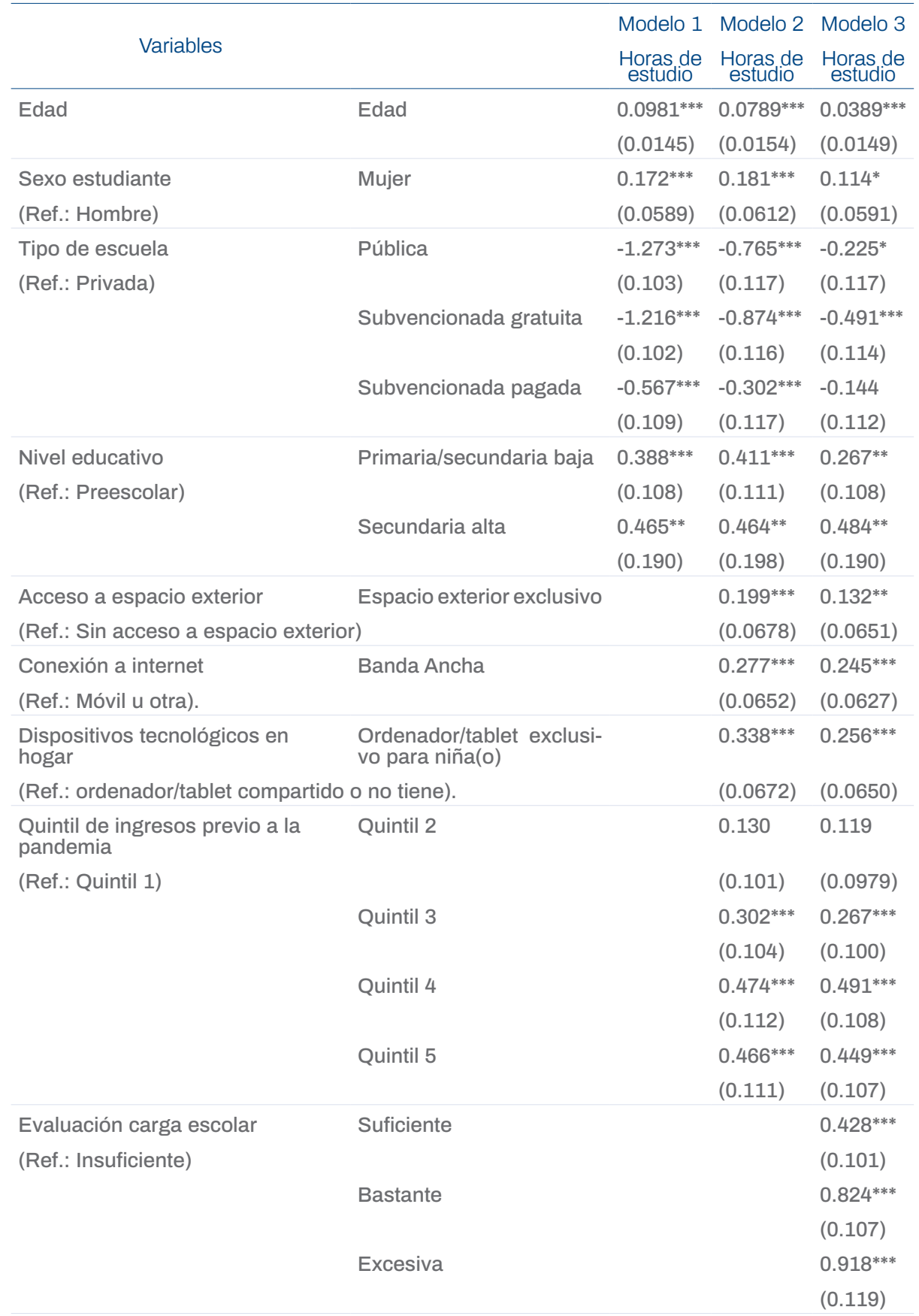




\begin{tabular}{|c|c|c|c|c|}
\hline \multirow{2}{*}{ Variables } & & \multirow{2}{*}{$\begin{array}{l}\text { Modelo } 1 \\
\text { Horas de } \\
\text { estudio }\end{array}$} & \multirow{2}{*}{$\begin{array}{c}\text { Modelo } 2 \\
\text { Horas de } \\
\text { estudio }\end{array}$} & \multirow{2}{*}{$\begin{array}{l}\text { Modelo } 3 \\
\text { Horas de } \\
\text { estudio }\end{array}$} \\
\hline & & & & \\
\hline $\begin{array}{l}\text { Frecuencia semanal de clases } \\
\text { online }\end{array}$ & Todos los días & & & $1.196 * * *$ \\
\hline \multirow[t]{5}{*}{ (Ref.: Nunca) } & & & & $(0.108)$ \\
\hline & Algunos días & & & $0.583^{* * *}$ \\
\hline & & & & $(0.104)$ \\
\hline & Un día & & & $0.304^{* *}$ \\
\hline & & & & $(0.124)$ \\
\hline Problemas socioemocionales & $\begin{array}{l}\text { Le cuesta concentrarse, a } \\
\text { menudo o siempre. }\end{array}$ & & & $-0.328 * * *$ \\
\hline \multirow[t]{7}{*}{ (Ref.: Nunca o pocas veces) } & & & & $(0.0680)$ \\
\hline & $\begin{array}{l}\text { No quiere hacer las tareas, } \\
\text { a menudo o siempre. }\end{array}$ & & & $-0.331^{* * *}$ \\
\hline & & & & $(0.0694)$ \\
\hline & Constante & $2.765^{* * *}$ & $1.796^{* * *}$ & $0.959^{* * *}$ \\
\hline & & $(0.115)$ & $(0.159)$ & $(0.199)$ \\
\hline & $\mathrm{N}$ & 4.229 & 3.856 & 3.856 \\
\hline & R2 & 0.114 & 0.138 & 0.216 \\
\hline
\end{tabular}

Errores estándar entre paréntesis / ${ }^{* * *} p<0.01,{ }^{* *} p<0.05,{ }^{*} p<0.1$

Fuente: elaboración propia

\section{Discusión}

En el presente artículo se han explorado las condiciones de aprendizaje de los escolares y sus familias en este contexto de crisis. Tal como lo pronosticaban los estudios en otras naciones (Bonal y González, 2020) se observaron importantes desigualdades en el acceso a oportunidades de aprendizaje: el porcentaje de alumnos que no tuvieron "Nunca" acceso a la educación online fue mayor aún al 10\% encontrado por Reimers y Schleicher (2020). Esto es especialmente relevante a la luz de los resultados de las regresiones, donde los estudiantes que "Nunca" recibieron clases online dedicaron un promedio de 72 minutos menos al día a actividades de aprendizaje que sus pares.

Como hemos divisado en el análisis, las desigualdades por tipo de escuela tienen un origen anterior a esta, específicamente en la estratificación socioeconómica de las escuelas, donde la mayor vulnerabilidad de las familias de los estudiantes de escuelas gratuitas se relaciona directamente con una escasez de recursos para el aprendizaje. Esta escasez se observa tanto en el acceso dispositivos tecnológicos, conexión a internet, un escritorio o habitación propia para estudiar, que afectaron también de forma significativa el uso del tiempo para el aprendizaje en pandemia según los resultados de las regresiones (Filandri y Olagnero, 2014; Dietrich, Patzina y Lerche, 2021).

Los resultados indicaron también que las experiencias de aprendizaje en pandemia tienen un componente relevante de género, tanto de parte de progenitores como de estudiantes. Así, las mujeres a cargo de los hogares han percibido una ma- 
yor carga de las tareas domésticas y de cuidados, recayendo tanto en adultas como niñas, lo cual se ve reafirmado por los hallazgos que presentan a la madre como el principal pilar de apoyo de la escuela en casa. Además, hay diferencias por género en las actividades que desarrollan niñas y niños en casa, teniendo las actividades femeninas mayor diversidad, menor conexión con pantallas y ser de un carácter más sociable, creativo y colaborativo. Es destacable también que, aún controlado por las variables del hogar y de la escuela, las estudiantes mujeres presenten una ventaja en el número de horas de estudio diarias, en comparación a sus pares masculinos. Si bien se indagó indirectamente acerca de que tan de acuerdo estaban con la afirmación de si las niñas y mujeres están expuestas a distintos tipos de violencia de género, los altos índices en la respuesta reflejan que ellas corren un mayor riesgo al encontrarse solas en casa.

Roe, et al. (2021) reportaron una relación entre las rutinas y actividades de alumnos y su bienestar físico y emocional, como la actividad física, en nuestro análisis encontramos niveles altos de sedentarismo entre las y los estudiantes chilenos, lo cual se agrava entre aquellos que residen en viviendas sin espacios exteriores de uso exclusivo que, a su vez, son más frecuentes en los hogares con mayor número de metros cuadrados y, predeciblemente, mejor nivel socioeconómico. La carencia de acceso a espacios exteriores también se relacionó con peores resultados socioemocionales durante la pandemia y un menor tiempo dedicado al estudio formal.

A su vez, darle la justa importancia al bienestar socioemocional de los estudiantes es clave, como investigó Tanyu, et al. (2020), y como también comprobamos en nuestro estudio, la falta de socialización, en momento de crisis, aumenta la vulnerabilidad en la salud mental de niñas y niños, lo que podría tener fuertes repercusiones en un futuro, si es que no nos responsabilizamos de ese plano de la vida. Emociones como la ansiedad, miedos y desgano son repetitivas y transversales a nivel mundial en los hogares y familias, y da cuenta de los desafíos futuros en cómo construiremos la educación con un carácter más integral.

En ese sentido, como hemos visto, la cadena que conforma la educación en casa en un contexto de pandemia es frágil, porque son muchos los puntos de quiebre y cualquiera de ellos compromete buena parte o la totalidad del proceso. No basta con que los docentes preparen buenas clases online, si la conexión a internet de los alumnos no les permite verlas de manera fluida; de poco sirven tablets y ordenadores, si no hay un espacio tranquilo para usarlo; poco importa que esté todo bien dispuesto, si el ánimo no alcanza para salir de la cama. La escuela pandémica pende de un hilo cotidianamente. Es decir, al recluir la escuela a la casa, la experiencia educativa de la niña o niño ha dependido necesariamente de sus condiciones de hogar. En ese sentido, el compromiso colectivo actual implica proveer a cada niño o niña una experiencia educacional independiente de sus privilegios o desventajas, que los entienda como sujetos de derecho, y que, al cierre de las escuelas, los costos sean lo menos injustos posible.

Del mismo modo, se debe actuar interdependientemente y como comunidad, asegurar el derecho a la educación de los más vulnerables implica comprender que nadie estará seguro hasta que aquellos a quienes les es más difícil asegurar sus condiciones de vida, también estén seguros (UNICEF España, 2020). La necesidad 
de repensar lo urgente, deseable y posible en torno a las fragilidades de la escuela pandémica, se da a diferentes escalas, por lo que la inversión de los esfuerzos es a nivel colectivo y debe ser traducida en oportunidades de aprendizaje que devuelvan el sentido de una educación integral basada en la justicia social.

\section{Agradecimientos}

Agradecemos el financiamiento de este estudio a la Agencia Nacional de Investigación y Desarrollo de Chile (ANID), obtenido a través del Concurso para la Asignación Rápida de Recursos Para Proyectos de Investigación sobre el Coronavirus (COVID-19). También se agradece el financiamiento otorgado por ANID/PIA/Fondos Basales para Centros de Excelencia FB0003.

\section{Referencias}

Andrew, A., Cattan, S., Costa Dias, M., Farquharson, C., Kraftman, L., Krutikova, S., . . Sevilla, A. (2020). Learning during the lockdown: real-time data on children's experiences during home learning. London: Institute for Fiscal Studies. Recuperado de https://bit.ly/3g13Be5

Asanov, I., Flores, F., McKenzie, D., Mensmann, M. y Schulte, M. (2020). Remote-learning, Time-Use, and Mental Health of Ecuadorian High-School Students during the COVID-19 Quarantine. Policy Research Working Paper 9252, 1-23. Recuperado de https://bit.ly/3fXHiWJ

Azevedo, J., Hasan, A., Goldemberg, D. y Aroob, S. (2020). Simulating the Potential Impacts of COVID-19 School Closures on Schooling and Learning Outcomes. A set of Global Estimates. Policy Research Working Paper(9284), 1-58. Recuperado de https://bit.ly/3fX2A6Z

Baez, J. y Santos, I. (2007). Children's Vulnerability to Weather Shocks: A Natural Disaster as a Natural Experiment. New York: Social Science Research Network. Recuperado de https:// bit.ly/3vSaRQx

Bellei, C. (2013). El estudio de la segregación socioeconómica y académica de la educación chilena. Estudios Pedagógicos XXXIX(1), 325-345.

Bonal, X. y González, S. (2020). Educación formal e informal en confinamiento: una creciente desigualdad de oportunidades de aprendizaje. Revista de Sociología de la Educación (RASE), 44-62. https://doi.org/10.7203/RASE.14.1.18177

Cabrera, L., Pérez, C. y Santana, F. (2020). ¿Se Incrementa la Desigualdad de Oportunidades Educativas en la Enseñanza Primaria con El Cierre Escolar por el Coronavirus? International Journal of Sociology of Education, Special Issue: COVID-19 Crisis and Socioeducative Inequalities and Strategies to Overcome them, 27-52. Recuperado de https://bit.ly/3caxPu7

Carroll, N., Sadowski, A., Laila, A., Hruska, V., Nixon, M., Ma, D. y Haines, J. (2020). The Impact of COVID-19 on Health Behavior, Stress, Financial and Food Security among Middle to High Income Canadian Families with Young Children. Nutrients, 12(8), 1-14. https://doi. org/10.3390/nu12082352

Cauchemez, S., Ferguson, N., Wachtel, C., Tegnell, A., Saour, G., Duncan, B. y Nicoll, A. (2009). Closure of schools during an influenza pandemic. The Lancet Infectious Diseases, 473481. Recuperado de https://bit.ly/3idıOHf

Dietrich, H., Patzina, A. y Lerche, A. (2021). Social inequality in the homeschooling efforts of German high school students during a school closing period. European Societies, S348-S369. Recuperado de https://doi.org/10.1080/14616696.2020.1826556

Filandri, M. y Olagnero, M. (2014). Housing Inequality and Social Class in Europe. Housing Studies, 977-993. https://doi.org/10.1080/02673037.2014.925096 
Fontenelle-Tereshchuk, D. (2020). Mental Health and the COVID-19 Crisis: The Hopes and Concerns for Children as Schools Re-open. Interchange. https://doi.org/10.1007/s10780020-09413-1

Fontenelle-Tereshchuk, D. (2021). 'Homeschooling' and the COVID-19 Crisis: The Insights of Parents on Curriculum and Remote Learning. Interchange. https://doi.org/10.1007/s10780-02109420-w

Giannini, S. y Albrectsen, A.-B. (2020, marzo 31). El cierre de escuelas debido a la Covid-19 en todo el mundo afectará más a las niñas. UNESCO. Recuperado de https://bit.ly/2SOLTT9

Hanusheck, E. y Woessmann, L. (2020). The economic impacts of learning losses. OECD Education Working Papers(225), 1-23. https://doi.org/10.1787/21908d74-en

Hortigüela-Alcalá, D., Pérez-Pueyo, Á., López-Aguado, M., Manso-Ayuso, J. y Fernández-Río, J. (2020). Familias y Docentes: Garantes del Aprendizaje durante el confinamiento. Revista Internacional de Educación para la Justicia Social, 353-370. https://doi.org/10.15366/ riejs2020.9.3.019

livari, N., Sharma, S. y Ventä-Olkkonen, L. (2020). Digital transformation of everyday life - How COVID-19 pandemic transformed the basic education of the young generation and why information management research should care? International Journal of Information Management, 55. https://doi.org/10.1016/j.ijinfomgt.2020.102183

Mineduc (2020). Resumen Estadístico de la Educación Preliminar 2020. Centro de Estudios Mineduc (CEM), Santiago, Chile.

Reimers, F. y Schleicher, A. (2020). Aprendiendo durante la pandemia. De la disrupción a la innovación. Cambridge: OECD. Global Education Innovation. Recuperado de https://bit.ly/3c8Z7RI

Roe, A., Blikstad-Balas, M. y Pedersen, C. (2021). The Impact of COVID-19 and Homeschooling on Students' Engagement With Physical Activity. Frontiers in sports and active living, 2. https:// doi.org/10.3389/fspor.2020.589227

Tanyu, M., Spier, E., Pulizzi, S., Rooney, M., Sorenson, I. y Fernandez, J. (2020). Improving Education Outcomes for Students Who Have Experienced Trauma and/or Adversity. Paris: OECD Education Working Paper No. 242. https://doi.org/10.1787/54d45980-en

Tarabini, A. (2020). ¿Para qué sirve la escuela? Reflexiones sociológicas en tiempos de pandemia global. Revista de Sociología de la Educación, 145-155. Recuperado de https://bit.ly/3yVcWg6

UNESCO. (2017). Guía para asegurar la inclusión y la equidad en la educación. París. Recuperado de https://bit.ly/3fEMVKB

UNESCO (2020). Adverse consequences of school closures. Recuperado de https://bit.ly/2Txsp65

UNICEF. (2015). El ébola: llegar a cero - para las comunidades, para los niños y las niñas, para el futuro. Dakar. Recuperado de https://uni.cf/3vGXFhc

UNICEF. (2020, septiembre 23). Mientras las aulas permanecen cerradas para la mitad de los escolares del mundo, UNICEF y la Unión Europea instan a los países a dar prioridad a las escuelas. Recuperado de https://bit.ly/3vLxsy8

UNICEF España. (2020, Abril). La Educación frente al COVID-19. Propuestas para impulsar el derecho a la educación durante la emergencia. Recuperado de https://bit.ly/34F6Xhy

Van der Vlies, R. (2020). Digital strategies in education across OECD countries: Exploring education policies on digital technologies. OECD Education Working Papers No. 226. doi:https:// dx.doi.org/10.1787/33dd4c26-en

Zhao, Y., Guo, Y., Xiao, Y., Zhu, R., Sun, W., Huang, W., . . Wu, J.-L. (2020). The Effects of Online Homeschooling on Children, Parents, and Teachers of Grades 1-9 During the COVID-19 Pandemic. Medical Science Monitor, 1-10. Recuperado de https://bit.ly/3p7b8w4

Cómo citar es artículo en APA

Ponce Mancilla, T., Vielma Hurtado, C. y Bellei Carvacho, C. (2021). Experiencias educativas de niñas, niños y adolescentes chilenos confinados por la pandemia COVID-19. Revista Iberoamericana de Educación, 86(1), 97-115. https://doi.org/10.35362/rie8614415 